\title{
A jet-like outflow toward the high-mass (proto) stellar object IRAS 18566+0408
}

\author{
Q. Zhang ${ }^{1}$, T. K. Sridharan ${ }^{1}$, T. R. Hunter ${ }^{2}$, Y. Chen $^{1}$, H. Beuther ${ }^{3}$, and F. Wyrowski ${ }^{4}$ \\ 1 Harvard-Smithsonian Center for Astrophysics, 60 Garden Street, Cambridge, Massachusetts 02138, USA \\ e-mail: qzhang@cfa.harvard.edu \\ 2 National Radio Astronomical Observatory, 520 Edgemont Road, Charlottesville, VA 22903-2475, USA \\ 3 Max-Planck-Institute for Astronomy, Königstuhl 17, 69117 Heidelberg, Germany \\ ${ }^{4}$ Max-Planck-Institut für Radioastronomie, Auf dem Hugel 69, 53121 Bonn, Germany
}

Received 12 January 2007 / Accepted 11 April 2007

\section{ABSTRACT}

\begin{abstract}
Context. Studies of high-mass protostellar objects reveal important information regarding the formation process of massive stars. Aims. We study the physical conditions in the dense core and molecular outflow associated with the high-mass protostellar candidate IRAS $18566+0408$ at high angular resolution.

Methods. We performed interferometric observations in the $\mathrm{NH}_{3}(J, K)=(1,1),(2,2)$ and $(3,3)$ inversion transitions, the $\mathrm{SiO} J=2-1$ and $\mathrm{HCN} J=1-0$ lines, and the 43 and $87 \mathrm{GHz}$ continuum emission using the VLA and OVRO.

Results. The $87 \mathrm{GHz}$ continuum emission reveals two continuum peaks MM-1 and MM-2 along a molecular ridge. The dominant peak MM-1 coincides with a compact emission feature at $43 \mathrm{GHz}$, and arises mostly from the dust emission. For dust emissivity index $\beta$ of 1.3, the masses in the dust peaks amount to $70 M_{\odot}$ for MM-1, and $27 M_{\odot}$ for MM-2. Assuming internal heating, the central luminosities of MM- 1 and MM-2 are $6 \times 10^{4}$ and $8 \times 10^{3} L_{\odot}$, respectively.

The $\mathrm{SiO}$ emission reveals a well collimated outflow emanating from MM-1. The jet-like outflow is also detected in $\mathrm{NH}_{3}$ at velocities similar to the $\mathrm{SiO}$ emission. The outflow, with a mass of $27 M_{\odot}$, causes significant heating in the gas to temperatures of $70 \mathrm{~K}$, much higher than the temperature of $<15 \mathrm{~K}$ in the extended core. Compact $\left(<3^{\prime \prime}\right)$ and narrow line $\left(<1.5 \mathrm{~km} \mathrm{~s}^{-1}\right) \mathrm{NH}_{3}(3,3)$ emission features are found associated with the outflow. They likely arise from weak population inversion in $\mathrm{NH}_{3}$ similar to the maser emission. Toward MM-1, there is a compact $\mathrm{NH}_{3}$ structure with a linewidth that increases from $5.5 \mathrm{~km} \mathrm{~s}^{-1} F W H M$ measured at $3^{\prime \prime}$ resolution to $8.7 \mathrm{~km} \mathrm{~s}^{-1}$ measured at $1^{\prime \prime}$ resolution. This linewidth is much larger than the $F W H M$ of $<2 \mathrm{~km} \mathrm{~s}^{-1}$ in the entire core, and does not appear to originate from the outflow. This large linewidth may arise from rotation/infall, or relative motions of unresolved protostellar cores.
\end{abstract}

Key words. ISM: kinematics and dynamics - ISM: H II regions - ISM: clouds - masers - ISM: jets and outflows - stars: formation

\section{Introduction}

Systematic surveys in the past decade identified hundreds of high-mass protostellar candidates (Molinari et al. 1996; Sridharan 2002; Fontani et al. 2005). These objects, selected initially from the IRAS point source catalog, typically have far infrared luminosities of $>10^{3} L_{\odot}$, contain $10^{2}-10^{4} M_{\odot}$ of dense molecular gas (Molinari et al. 2002; Beuther et al. 2002a; Williams et al. 2004; Beltrán et al. 2006), and are associated with massive molecular outflows (Zhang et al. 2001, 2005; Beuther et al. 2002b). Compared with ultra compact HII (UCHII) regions, high-mass protostellar candidates have similar amounts of dense molecular gas, but are less luminous and have much weaker emission at centimeter wavelengths. Therefore, they are likely to be in an earlier evolutionary stage than the UCHII phase.

These surveys were carried out mostly using single dish telescopes with angular resolutions of $>10^{\prime \prime}$. High angular resolution imaging is required to probe dense cores and molecular outflows at spatial scales relevant to massive protostars. In the past few years, images from (sub)mm interferometers have often resolved poorly-collimated outflows identified by single dish telescopes into multiple well-collimated outflows (e.g. IRAS 05358+3543, Beuther et al. 2002d; AFGL 5142, Zhang et al. 2007). In the meantime, high resolution images in $\mathrm{NH}_{3}$ and other dense molecular gas tracers reveal interesting kinematics close to massive protostars (Zhang et al. 1998, 2002).

In this paper, we present a high resolution study toward the high-mass protostellar candidate IRAS $18566+0408$. At a kinematic distance of $6.7 \mathrm{kpc}$ (Sridharan et al. 2002), the source has a far infrared luminosity of several $10^{4} L_{\odot}$. The object was initially undetected at 2 and $6 \mathrm{~cm}$ at an rms of $0.16 \mathrm{mJy}$ and $0.1 \mathrm{mJy}$, respectively (Miralles et al. 1994), but later detected at $3.6 \mathrm{~cm}$ at a flux density of $0.7 \mathrm{mJy}$ (Carral et al. 1999), and at $2 \mathrm{~cm}$ at a flux of $0.7 \mathrm{mJy}$ (Araya et al. 2005).

This region is associated with $\mathrm{H}_{2} \mathrm{O}$ maser emission at $22 \mathrm{GHz}, \mathrm{CH}_{3} \mathrm{OH}$ maser emission at $6.7 \mathrm{GHz}$, and $\mathrm{H}_{2} \mathrm{CO}$ maser emission at $8 \mathrm{GHz}$ (Miralles et al. 1994; Slysh et al. 1999; Beuther et al. 2002c; Araya et al. 2005). Dense gas traced by $\mathrm{CS}$ and $\mathrm{CH}_{3} \mathrm{CN}$, as well as (sub)mm continuum emission is observed (Bronfman et al. 1996; Sridharan et al. 2002; Beuther et al. 2002a; Williams et al. 2004). $\mathrm{NH}_{3}(1,1)$ and $(2,2)$ emission was detected first by Miralles et al. (1994), and also by Molinari et al. (1996) (source 83) and Sridharan et al. (2002) with single dish telescopes.

Beuther et al. (2002b) report a CO outflow in the northwestsoutheast direction. The geometric center of the outflow, however, is about $10^{\prime \prime}$ north of the $1.2 \mathrm{~mm}$ emission peak. Since the $1.2 \mathrm{~mm}$ continuum position is consistent with that of the submm 
Table 1. List of observational parameters.

\begin{tabular}{llllll}
\hline \hline Instrument & $\begin{array}{l}\text { Date of } \\
\text { Observations }\end{array}$ & Line & $\begin{array}{l}\text { Bandwidth } \\
(\mathrm{MHz})\end{array}$ & $\begin{array}{l}\text { Spectral } \\
\text { Res. }\left(\mathrm{km} \mathrm{s}^{-1}\right)\end{array}$ & $\begin{array}{l}\text { Integration } \\
\text { Time }(\mathrm{h})\end{array}$ \\
\hline VLA-CnB & $2001 / 07 / 23$ & $\mathrm{NH}_{3}(1,1),(2,2)$ & 3.12 & 0.6 & 0.7 \\
VLA-DnC & $2001 / 10 / 01$ & $\mathrm{NH}_{3}(3,3)$ & 3.12 & 0.3 & 1.0 \\
VLA-CnB & $2002 / 09 / 26$ & $\mathrm{NH}_{3}(1,1),(2,2)$ & 3.12 & 0.6 & 1.2 \\
VLA-CnB & $2002 / 09 / 26$ & $\mathrm{NH}_{3}(3,3)$ & 3.12 & 0.6 & 1.0 \\
VLA-CnB & $2002 / 09 / 26$ & $23 \mathrm{GHz}$ & 25 & - & 1.0 \\
VLA-DnC & $2003 / 01 / 16$ & $\mathrm{NH}_{3}(1,1),(2,2)$ & 3.12 & 0.3 & 0.6 \\
VLA-DnC & $2003 / 01 / 24$ & $\mathrm{NH}_{3}(3,3)$ & 3.12 & 0.6 & 1.0 \\
VLA-DnC & $2003 / 02 / 04$ & $43 \mathrm{GHz}$ & 50 & - & 2 \\
\hline OVRO-E & $2002 / 11 / 02$ & $\mathrm{HCN}(1,0)$ & 30 & 3.4 & 4.0 \\
OVRO-E & $2002 / 11 / 02$ & $\mathrm{SiO}(2-1)_{0}$ & 30 & 1.7 & 4.0 \\
OVRO-E & $2002 / 11 / 02$ & $\mathrm{HCO}^{+}(1-0)$ & 7.5 & 0.84 & 4.0 \\
OVRO-E & $2002 / 11 / 02$ & $87 \mathrm{GHz}$ & 4000 & - & 4.0 \\
OVRO-H & $2002 / 12 / 01$ & $87 \mathrm{GHz}$ & 4000 & - & 2.0 \\
\hline
\end{tabular}

emission (Williams et al. 2004), this offset is possibly caused by pointing problems in the $\mathrm{CO}$ observations with the IRAM 30 telescope (Beuther H., private communication). The $1.2 \mathrm{~mm}$ emission shows an extension of $15^{\prime \prime}$ toward the northwest of the peak emission. $\mathrm{SiO} J=2-1$ emission is detected in the region with a linewidth of $30 \mathrm{~km} \mathrm{~s}^{-1}$ at zero intensity (Beuther H., private communication).

The high resolution observations with the VLA and OVRO in this paper reveal a collimated outflow in $\mathrm{SiO}$ and kinematics in the dense core. In Sect. 2, we describe details of observations. In Sect. 3, we present the main observational results. In Sect. 4, we discuss the different kinematic components in the region. A summary is given in Sect. 5 .

\section{Observations}

\subsection{VLA}

\subsection{1. $\mathrm{NH}_{3}$ observations}

The VLA ${ }^{1}$ observations of IRAS $18566+0408$ were first conducted on 2001 July 23 in the $\mathrm{NH}_{3}(J, K)=(1,1)$ and $(2,2)$ lines in the $\mathrm{C}$ configuration. To improve the $\mathrm{S} / \mathrm{N}$ in the data, follow-up observations were made from 2001 October to 2003 January, in both $\mathrm{CnB}$ and DnC configurations in the $\mathrm{NH}_{3}(1,1),(2,2)$ and $(3,3)$ inversion transitions. The integration time on source was typically less than $1 \mathrm{~h}$ for each line. The pointing center of the observations was RA $(2000)=18^{\mathrm{h}} 59^{\mathrm{m}} 09^{\mathrm{s}} .88$ and Dec $(2000)=$ $+04^{\circ} 12^{\prime} 13^{\prime \prime}$. 6 . We used 1849+005, 3C 286 and 3C 273 as the gain, flux, and bandpass calibrators. The detailed parameters of the observations are summarized in Table 1.

The visibility data were calibrated using the NRAO Astronomical Image Processing System (AIPS). The uncertainty in the flux calibration is about $10 \%$. The calibrated visibilities from different epochs were combined for the same line and imaged in MIRIAD. The rms noise in the $(1,1),(2,2)$ and the $(3,3)$ lines is about 2 mJy in a $3^{\prime \prime}$ to $4^{\prime \prime}$ synthesized beam per $0.6 \mathrm{~km} \mathrm{~s}^{-1}$ wide channel.

\subsection{2. $23 \mathrm{GHz}$ and $43 \mathrm{GHz}$ continuum}

The continuum observations at $23 \mathrm{GHz}$ and $43 \mathrm{GHz}$ were carried out with the VLA on 2002 September 26 and 2003 February 04,

1 The National Radio Astronomy Observatory is operated by Associated Universities, Inc., under cooperative agreement with the National Science Foundation. respectively. At $43 \mathrm{GHz}$, we used the fast switching calibration scheme that alternated between IRAS18566+0408 and the gain calibrator $1849+005$ in a cycle of $2 \mathrm{~min}$. The total on-source time for IRAS $18566+0408$ was about $2 \mathrm{~h}$ at $43 \mathrm{GHz}$, and $1 \mathrm{~h}$ at $23 \mathrm{GHz}$. Calibration and imaging were performed in AIPS. The flux calibration was done by comparing to $3 \mathrm{C} 286$. The absolute flux scales are accurate to about $10 \%$. The rms is $0.1 \mathrm{mJy}$ in the $43 \mathrm{GHz}$ image, and $0.14 \mathrm{mJy}$ in the $23 \mathrm{GHz}$ image, respectively.

\subsection{OVRO}

The OVRO observations of IRAS $18566+0408$ were carried out during 2002 November to December. The $\mathrm{SiO} J=2-1(v=0)$, $\mathrm{HCN} J=1-0$ and $\mathrm{HCO}^{+} J=1-0$ lines were observed simultaneously in the lower sideband, along with $87 \mathrm{GHz}$ continuum. The $\mathrm{SiO}$ line was observed with a total bandwidth of $31 \mathrm{MHz}$ and a spectral resolution of $0.5 \mathrm{MHz}\left(1.7 \mathrm{~km} \mathrm{~s}^{-1}\right)$. The HCN line was observed with a bandwidth of $30 \mathrm{MHz}$ at a resolution of $1 \mathrm{MHz}\left(3.4 \mathrm{~km} \mathrm{~s}^{-1}\right)$. The $\mathrm{HCO}^{+}$line was observed with a bandwidth of $7.5 \mathrm{MHz}$ at a resolution of $0.25 \mathrm{MHz}\left(0.8 \mathrm{~km} \mathrm{~s}^{-1}\right)$. In addition, the analog correlator provided continuum measurements of $4 \mathrm{GHz}$ bandwidth. The pointing center of the OVRO observations was the same as that of the VLA observations. The detailed parameters of the observations are summarized in Table 1.

The visibility data were calibrated in the OVRO MMA package and exported to MIRIAD for imaging. The rms is $17 \mathrm{mJy}$ per $1.7 \mathrm{~km} \mathrm{~s}^{-1}$ channel for the line images, and $0.5 \mathrm{mJy}$ for the continuum. The $\mathrm{HCO}^{+}$emission is extended and suffers missing short spacing fluxes, thus, is not presented in this paper.

\section{Results}

\subsection{Continuum emission}

No emission is detected at $23 \mathrm{GHz}$ at a $1 \sigma \mathrm{rms}$ of $0.14 \mathrm{mJy}$. Figure 1 presents images of continuum emission at $43 \mathrm{GHz}$ (or $7.0 \mathrm{~mm}$ ) and $87 \mathrm{GHz}$ (or $3.4 \mathrm{~mm}$ ). The $43 \mathrm{GHz}$ emission shows a compact feature with the peak position at RA $(2000)=$ $18^{\mathrm{h}} 59^{\mathrm{m}} 09^{\mathrm{s}} 99$, Dec $(2000)=04^{\circ} 12^{\prime} 15^{\prime \prime} \cdot 7$. The emission appears to be slightly resolved with the $2^{\prime \prime} .7 \times 11^{\prime \prime} .3$ beam at a position angle of $-7.6^{\circ}$. The peak and integrated flux densities are $1 \mathrm{mJy} / \mathrm{beam}$ and $1.7 \mathrm{mJy}$, respectively, with a $1 \sigma$ error of $0.1 \mathrm{mJy}$. The emission has an extension in the northeast-southwest direction, which appears to be different from the position angle of the beam. The deconvolved size of the emission is $2^{\prime \prime} .0 \times 1$.' 2 with a position 


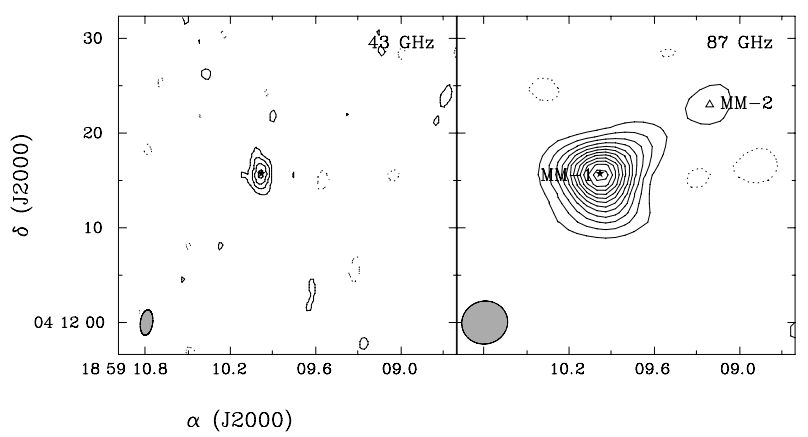

Fig. 1. Continuum emission at $43 \mathrm{GHz}$ and $87 \mathrm{GHz}$ toward IRAS $18566+0408$. The contour levels are in steps of $0.25 \mathrm{mJy} / \mathrm{beam}$ for the $43 \mathrm{GHz}$ continuum image, and $1.5 \mathrm{mJy} /$ beam for the $87 \mathrm{GHz}$ continuum image. The "star" symbol and "triangle" mark the continuum peaks MM-1 and MM-2, respectively. The size of the synthesized beam is marked by the shaded ellipse at the lower-left corner of each panel.

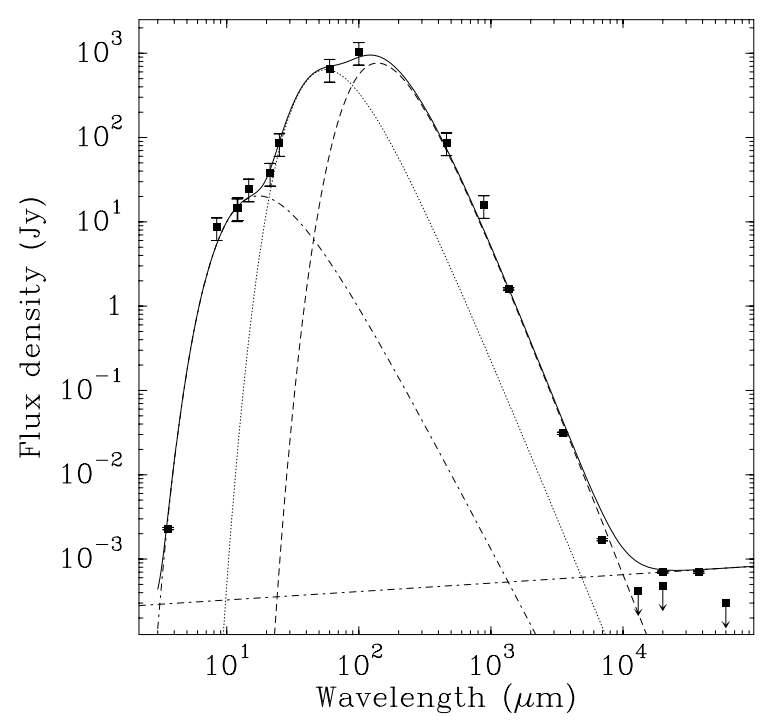

Fig. 2. The spectral energy distribution of IRAS $18566+0408$. The dashed line represents a cold dust component, the dotted line represents the warm dust component, the dash-dot line represents the freefree emission, and the solid line represents the sum of all three components.

angle of $24^{\circ}$. This corresponds to a size along the major axis of $1.3 \times 10^{4} \mathrm{AU}$.

The $87 \mathrm{GHz}$ emission is resolved with a 4 .' $9 \times 4$ ". 5 beam using natural weighting. The emission consists of a dominant peak, MM-1, coincident with the peak of the $43 \mathrm{GHz}$ emission to better than $0{ }^{\prime} 1$, and a secondary peak, MM-2, at RA (2000) = $18^{\mathrm{h}} 59^{\mathrm{m}} 09^{\mathrm{s}} .21$, Dec $(2000)=04^{\circ} 12^{\prime} 22^{\prime \prime} .6$. The peak flux density of MM-1 is $18 \mathrm{mJy} / \mathrm{beam}$, with an integrated flux density of $31 \mathrm{mJy}$. MM-2 is much weaker, with a peak flux density of $2.6 \mathrm{mJy} / \mathrm{beam}$. The uncertainty in these measurements is about $15 \%$. There appears to an extented filament at a position angle of $-58^{\circ}$ connecting MM- 1 and MM-2, which is better seen in the lower resolution (11") $1.2 \mathrm{~mm}$ map in Beuther et al. (2002a).

Figure 2 shows the spectral energy distribution of the continuum peak MM-1. The $1.3 \mathrm{~cm} 3 \sigma$ upper limit $(1 \sigma=0.14 \mathrm{mJy})$ is from this paper. The $3.6 \mathrm{~cm}$ continuum detection is from Carral et al. (1999). The $2 \mathrm{~cm}$ and $6 \mathrm{~cm}$ data are from Miralles et al. (1994) and Araya et al. (2005). The $1.2 \mathrm{~mm}, 850 \mu \mathrm{m}$ and $450 \mu \mathrm{m}$ measurements are from the IRAM 30-m telescope and JCMT (Beuther et al. 2002c; Williams et al. 2004). The mid to far infrared data are from IRAS, MSX and Spitzer IRAC measurements.

For emission at longer $\mathrm{cm}$ wavelengths, the contribution from dust is negligible. Toward MM-1, a faint continuum source was detected at $2 \mathrm{~cm}(0.7 \mathrm{mJy})$ by Araya et al. (2005), and at $3.6 \mathrm{~cm}$ (0.7 mJy) by Carral et al. (1999). However, we fail to detect the source at $1.3 \mathrm{~cm}$ at an angular resolution of $1^{\prime \prime}$ and a $1 \sigma \mathrm{rms}$ of $0.14 \mathrm{mJy}$. The detections by Araya et al. (2005) and Carral et al. (1999) were made at resolutions of a few arcseconds, and can be reconciled with the non detection in this paper if the source is more extended than $1^{\prime \prime}$. However, an inconsistency remains at $2 \mathrm{~cm}$ at which Miralles et al. (1994) failed to detect the source with a $1 \sigma \mathrm{rms}$ of $0.16 \mathrm{mJy}$ at a resolution of $5^{\prime \prime}$. A possible reconciliation is that the flux varies with time. Despite the apparent differences, the faintness of the $\mathrm{cm}$ emission indicates that the massive star in this region is still extremely young in its evolution, and has not produced significant free-free emission.

The measurements from wavelengths shortward of $1.2 \mathrm{~mm}$ have poorer spatial resolution and sample a much larger area in the region. We fit a greybody model to the entire spectral energy distribution from radio to infrared (IR) wavelengths. The far-IR measurements have a typical resolution of $\sim 1^{\prime}$. The overall spectral energy distribution can be fitted by three dust components, with temperatures of 210,58 and $30 \mathrm{~K}$, respectively. The total luminosity of the region determined mainly by mid to far IR data at $\sim 1^{\prime}$ resolution is $8 \times 10^{4} L_{\odot}$. The fluxes at the $\mathrm{mm}$ and submm wavelengths give a spectral index $\alpha$ of 3.9, defined as $F_{v} \propto v^{\alpha}$, or $\beta=1.9$.

For the compact continuum source MM-1, we use the high resolution $7 \mathrm{~mm}$ and $3 \mathrm{~mm}$ data to derive a power law index more appropriate for a mass estimate. To minimize the difference in beam size between the two frequencies, we image the $87 \mathrm{GHz}$ data with a uniform weighting of the visibilities and obtain a peak flux density of $10 \mathrm{mJy} /$ beam with a 3 .' $3 \times 2$ '. 0 beam. These two values (10 and $1.0 \mathrm{mJy} / \mathrm{beam})$ produce an upper limit to the spectral index $\alpha$ of 3.3, or an upper limit to the emissivity index $\beta$ of 1.3. Assuming that the dust reaches an equilibium with the gas through collision at this high density environment (Burke \& Hollenbach 1983), we approximate the dust temperature by the gas kinetic temperature of $80 \mathrm{~K}$ measured in $\mathrm{NH}_{3}$ (see Sects. 3.2, 3.3 and 4.1). For a dust opacity law $\kappa \propto v^{\beta}$, and $\kappa(250 \mu \mathrm{m})=$ $12 \mathrm{~cm}^{2} \mathrm{~g}^{-1}$ from Hildebrand (1983), we obtain a mass within the 4 ". $9 \times 4$ ". 5 beam $(\sim 30000 \mathrm{AU})$ of $70 M_{\odot}$ for $\beta=1.3$. This mass is a small fraction of the mass $\left(2 \times 10^{3} M_{\odot}\right)$ estimated from the $1.2 \mathrm{~mm}$ emission for the entire region (Beuther et al. 2002a).

For the continuum peak MM-2, the non detection at $7 \mathrm{~mm}$ gives a $3 \sigma$ upper limit of $0.3 \mathrm{mJy}$. This value and the peak flux density of $2.6 \mathrm{mJy} /$ beam at $87 \mathrm{GHz}$ yield a spectral index of $>3$. Using assumptions similar to those for MM-1, we estimate the mass in the MM- 2 core. With a temperature of $30 \mathrm{~K}$ derived from the $\mathrm{NH}_{3}$ emission, we obtain a mass of $27 M_{\odot}$ for $\beta=1.3$, and $13 M_{\odot}$ for $\beta=1$.

\subsection{Line emission}

Figure 3 presents the integrated emission of the $\mathrm{NH}_{3}(J, K)=$ $(1,1),(2,2),(3,3)$ lines obtained from the VLA, and the SiO $J=$ $2-1$ and HCN $J=1-0$ transitions obtained from OVRO. In the $\mathrm{NH}_{3}(1,1)$ and $(2,2)$ lines, there appears to be extended emission in the northwest-southeast direction over a scale of $40^{\prime \prime}$. This component is relatively cold as the $(2,2)$ emission is less extended than the $(1,1)$ line. MM-2 is associated with $\mathrm{NH}_{3}$ gas and lies in a molecular ridge connecting MM- 1 and MM- 2 that is also seen in the dust emission. In addition to the extended 


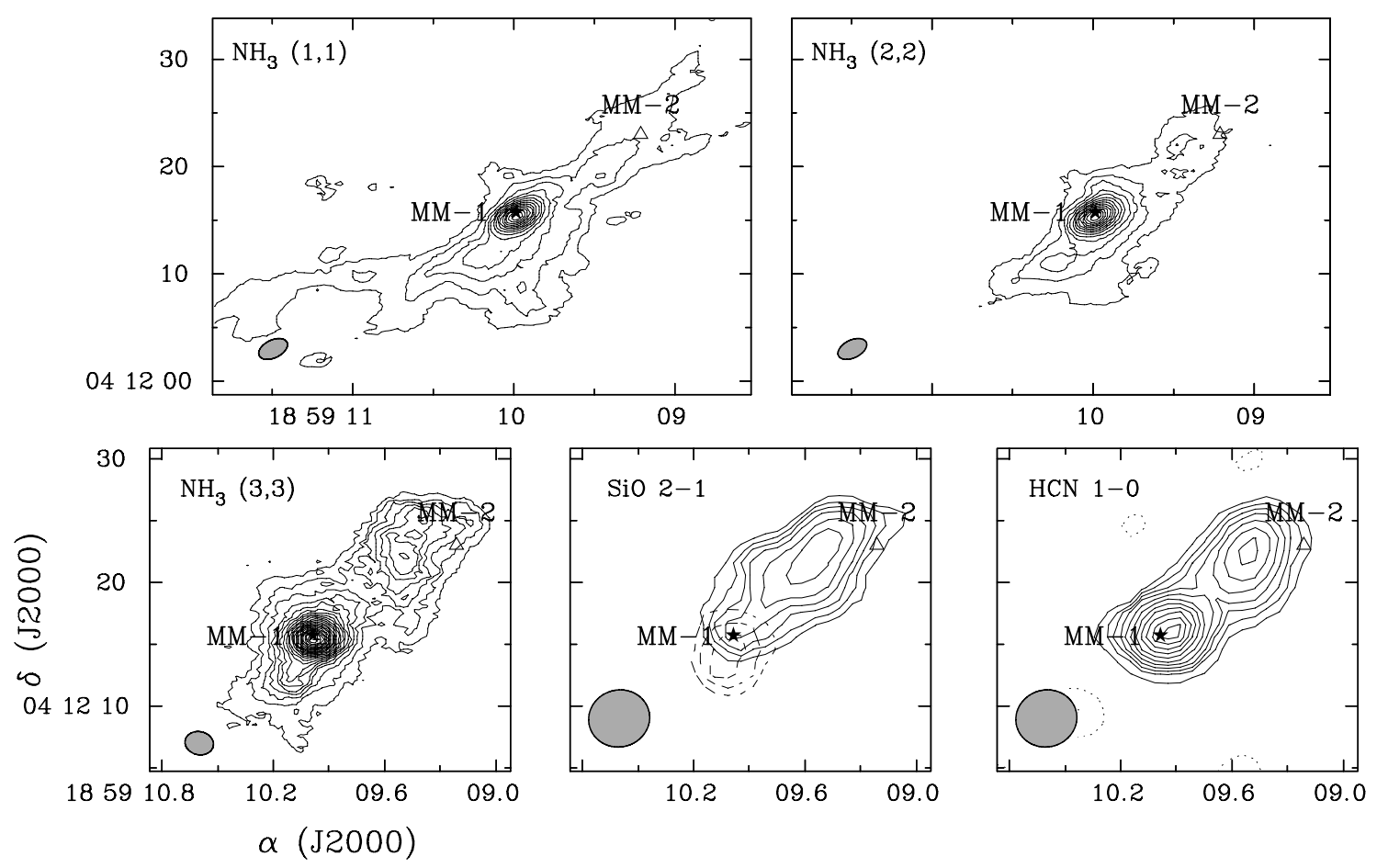

Fig. 3. The integrated emission of the $\mathrm{NH}_{3}(J, K)=(1,1),(2,2),(3,3), \mathrm{SiO} J=(2-1), \mathrm{HCN} J=(2-1)$ lines. The velocity range of the integration is 81 to $88 \mathrm{~km} \mathrm{~s}^{-1}$ for the $\mathrm{NH}_{3}(1,1)$ and $(2,2)$ lines. The $\mathrm{NH}_{3}(3,3)$ and $\mathrm{HCN}$ lines are integrated over the entire spectral line. For the $\mathrm{SiO}$ line, the blue-shifted emission (solid contours) is integrated from 50 to $83 \mathrm{~km} \mathrm{~s}^{-1}$ and the red-shifted emission (dashed contours) is integrated from 88 to $110 \mathrm{~km} \mathrm{~s}^{-1}$. The $\mathrm{NH}_{3}$ images are made from the VLA-D and C configuration data. The $\mathrm{SiO}$ and $\mathrm{HCN}$ data images are made from the OVRO E configuration data. The contour levels are in steps of $0.015 \mathrm{Jy} \mathrm{km} \mathrm{s}^{-1}$ for the $\mathrm{NH}_{3}(1,1)$ and $(2,2)$ lines, $0.02 \mathrm{Jy} \mathrm{km} \mathrm{s}^{-1}$ for the $\mathrm{NH}_{3}(3,3)$, $0.3 \mathrm{Jy} \mathrm{km} \mathrm{s}^{-1}$ for the SiO (2-1), and $0.6 \mathrm{Jy} \mathrm{km} \mathrm{s}^{-1}$ for the HCN (1-0). The "star" symbol and "triangle" mark the continuum peaks MM-1 and MM-2, respectively. The beam sizes are plotted at the lower-left corner of each panel.

gas component, a compact $\mathrm{NH}_{3}$ emission component associated with MM-1 is seen in all three $\mathrm{NH}_{3}$ lines, with a deconvolved size of 1 '. 2 . The peak intensity of the integrated emission in the $(2,2)$ line is $0.22 \mathrm{Jy} \mathrm{km} \mathrm{s}^{-1} /$ beam, similar to the value in the $(1,1)$ line. Thus the gas in this component is relatively warm. In the $\mathrm{NH}_{3}(3,3)$ line, there is also extended emission in the northwestsoutheast orientation. The extension, at a position angle of $135^{\circ}$, is similar to the emission in $\mathrm{SiO}$ and $\mathrm{HCN}$. Unlike in the case of the $\mathrm{NH}_{3}(1,1)$ and $(2,2)$ emission, MM-2 is located toward the edge of the $\mathrm{NH}_{3}(3,3)$, $\mathrm{SiO}$ and $\mathrm{HCN}$ emission.

To show detailed kinematics in the region, Fig. 4 presents the channel maps of the $\mathrm{NH}_{3}(J, K)=(1,1),(2,2)$ and $(3,3)$ lines. The extended emission is present mostly at velocities from 84 to $87 \mathrm{~km} \mathrm{~s}^{-1}$, with a peak velocity of $85.2 \mathrm{~km} \mathrm{~s}^{-1}$ corresponding to the cloud systemic velocity (Bronfman et al. 1996). The typical linewidth for the extended emission is about $1-2 \mathrm{~km} \mathrm{~s}^{-1}$ in $F W H M$ and the typical temperature is $<15 \mathrm{~K}$ (see the temperature map in Fig. 6 and discussions in Sect. 4). The relatively narrow linewidth and low temperature in the gas indicate that this extended component is from the quiescent gas in the core.

At velocities of 86 to $87 \mathrm{~km} \mathrm{~s}^{-1}$, there appears to be a molecular ridge (position angle of $148^{\circ}$ ) between MM-1 and MM-2 in the $(1,1)$ and $(2,2)$ emission. MM-2 coincides with a peak in the $\mathrm{NH}_{3}$ emission (see channel $86.4 \mathrm{~km} \mathrm{~s}^{-1}$ ). At velocities less than $84 \mathrm{~km} \mathrm{~s}^{-1}$ and greater than $87 \mathrm{~km} \mathrm{~s}^{-1}$, there appears to be compact $\mathrm{NH}_{3}$ emission toward the position of MM-1. This compact emission, with a $F W H M$ of $5.5 \mathrm{~km} \mathrm{~s}^{-1}$ measured at $3^{\prime \prime}$ resolution, is strong in the $(2,2)$ emission relative to the $(1,1)$ emission, indicating that the $\mathrm{NH}_{3}$ gas is rather warm. The ratio of the $\mathrm{NH}_{3}$ $(1,1)$ and $(2,2)$ lines gives a rotational temperature of $45 \mathrm{~K}$.
In the $\mathrm{NH}_{3}(3,3)$ line, the extended component seen in the $(1,1)$ and $(2,2)$ is not as dominant. A compact emission component toward the position of MM-1 stands out prominently. Since the $(3,3)$ transition has a higher upper energy level $(124 \mathrm{~K})$ as compared to the $(1,1)(23 \mathrm{~K})$ and $(2,2)(65 \mathrm{~K})$ lines, the $(3,3)$ emission confirms that the compact component is rather warm.

In addition to the compact component toward MM-1, there appear to be four additional compact emission components in the $\mathrm{NH}_{3}(3,3)$ line, two toward the east of MM-1 in the velocity channels of 84.6 and $85.5 \mathrm{~km} \mathrm{~s}^{-1}$, one to the west of MM-1 in the velocity channel of $85.2 \mathrm{~km} \mathrm{~s}^{-1}$, and one to the northwest of MM-1 from velocities of 85.5 to $85.8 \mathrm{~km} \mathrm{~s}^{-1}$. We refer to these features as "A", "B", "C" and "D", respectively (the crosses in the channel maps in Fig. 4). There appear to be no corresponding emission peaks in the $\mathrm{NH}_{3}(1,1)$ and $(2,2)$ lines. Unlike the broad $\mathrm{NH}_{3}(3,3)$ line emission toward MM-1, these four components have rather narrow velocity width of about $1.5 \mathrm{~km} \mathrm{~s}^{-1}$ in FWHM, but extended line wing emission. We will discuss these features further in Sect. 4.

Besides the compact emission components in Fig. 4c, the $\mathrm{NH}_{3}(3,3)$ emission also shows an extended structure in the southeast-northwest direction. This structure has rather broad line wings (15 $\mathrm{km} \mathrm{s}^{-1}$ from the cloud velocity), and high temperatures of $70 \mathrm{~K} . \mathrm{MM}-2$ is not associated with any peaks of the $(3,3)$ emission.

Figure $5 \mathrm{a}$ presents the channel maps of the $\mathrm{SiO} J=2-1$ transition. The $\mathrm{SiO}$ emission is elongated and lies mostly to the northwest of MM-1 at a position angle of $135^{\circ}$, similar to the extended emission in the $\mathrm{NH}_{3}(3,3)$ line. The $\mathrm{SiO}$ emission is present from velocities of 70 to $93 \mathrm{~km} \mathrm{~s}^{-1}$. There appears to 


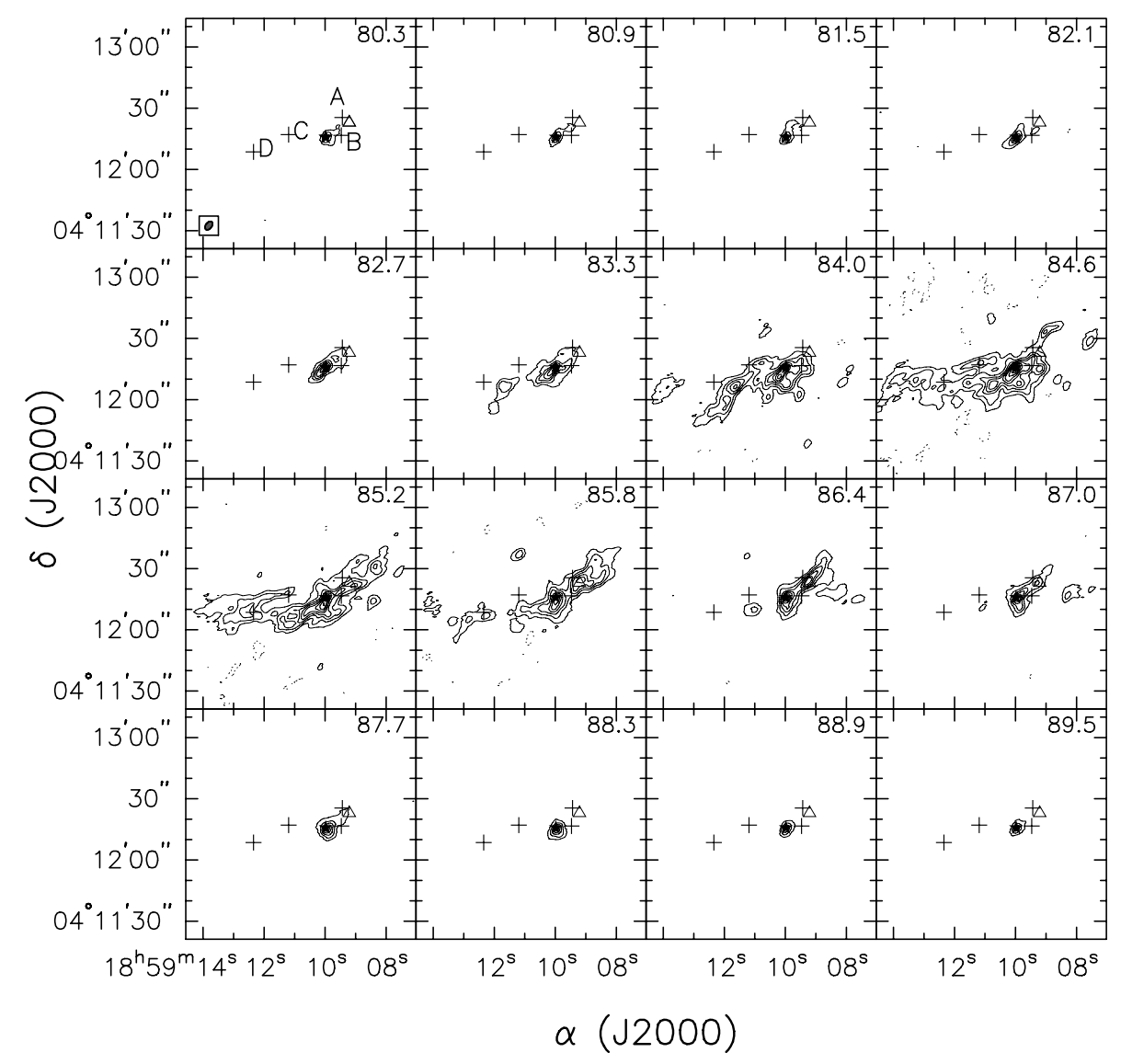

Fig. 4a. The channel maps of the $\mathrm{NH}_{3}(J, K)=(1,1)$ (Fig. 4a), $(2,2)$ (Fig. 4b) and (3, 3) (Fig. 4c) lines. The images were made using the VLA-D configuration data only. The contour levels are in steps of $8 \mathrm{mJy} /$ beam $(\sim 4 \sigma)$ for the $\mathrm{NH}_{3}(1,1)$ and $(2,2)$ lines and in steps of $9 \mathrm{mJy} /$ beam $(\sim 3 \sigma)$ for the $(3,3)$ line. The beam sizes are plotted at the lower-left of the first panel. The velocity of the channel is plotted at the upper-right of each panel. The $(1,1)$ and $(2,2)$ images have a spectral resolution of $0.6 \mathrm{~km} \mathrm{~s}^{-1}$, while the $(3,3)$ images have a spectral resolution of $0.3 \mathrm{~km} \mathrm{~s}^{-1}$. The "star" symbol and "triangle" mark the continuum peaks MM-1 and MM-2, respectively. The crosses mark the position of the hot $\mathrm{NH}_{3}(3,3)$ features.

be higher velocity $\mathrm{SiO}$ emission toward MM-1, but none toward $\mathrm{MM}-2$. Since $\mathrm{SiO}$ abundance is typically low in quiescent clouds (Ziurys et al. 1989) and is enhanced by a few orders of magnitude in outflows (e.g. Zhang et al. 1995) due to shock processes (Pineau des Forêts et al. 1997), the SiO emission here most likely traces a well collimated outflow originated from MM-1. The orientation of the $\mathrm{SiO}$ outflow is consistent with the bipolar $\mathrm{CO}$ outflow reported by Beuther et al. (2002).

Figure $5 \mathrm{~b}$ presents channel maps of the HCN 1-0 emission. The HCN 1-0 transition has three hyperfine components $(F=1-1,2-1$ and $0-1)$ at relative frequencies corresponding to $4.8,0$ and $-7.1 \mathrm{~km} \mathrm{~s}^{-1}$, respectively. We set the hyperfine component $F=2-1$ at a $V_{\mathrm{LSR}}$ of $85.2 \mathrm{~km} \mathrm{~s}^{-1}$, the cloud systemic velocity. It appears that the emission from the $F=1-1$ component is weak. The $F=2-1$ and $0-1$ components are detected around 80 and $73 \mathrm{~km} \mathrm{~s}^{-1}$, respectively (see also Fig. 7), $5 \mathrm{~km} \mathrm{~s}^{-1}$ blue shifted from the cloud velocity. The HCN emission arises mainly in two strong peaks: one is associated with the dust peak MM-1, the other is $4^{\prime \prime}$ offset from MM-2 and coincides with the $\mathrm{SiO}$ peak in the outflow. Little emission is detected toward MM-2.

The measured flux ratios of the three HCN hyperfine components $(F=0-1,2-1$ and $1-1)$ amount to $1: 1: 0.2$. Under the LTE condition, line ratios vary from 1:5:3 for optically thin emission to $1: 1: 1$ for optically thick emission. The measured ratios are not consistent with partially optically thick gas under LTE. Anomalous hyperfine ratios of $\mathrm{HCN}$ have been found in both cold dark clouds and warm clouds around HII regions (Walmsley et al. 1982; Cernicharo et al. 1984). Possible causes involve overlapping hyperfine components of higher rotational transitions or core-envelope density structures/velocity gradients in the cloud (Gonzalez-Alfonso \& Cernicharo 1993). The ratios measured in IRAS $18566+0408$ are different from those in dark or warm clouds. Missing short spacing flux in the interferometer data can affect the observed core and envelope emissions differently, which in turn affects the hyperfine ratios. Therefore, we do not further investigate this issue quantitatively.

\subsection{Rotational temperature}

We derive rotational temperatures of the $\mathrm{NH}_{3}$ gas. In the calculation, we assumed LTE conditions in the gas and followed the procedure outlined in Ho \& Townes (1983). Figure 6 presents a map of rotational temperature derived from the $\mathrm{NH}_{3}(1,1)$ and $(2,2)$ lines. In most of the core, temperatures are around $10 \mathrm{~K}$ to $15 \mathrm{~K}$. Higher rotational temperatures of about $45 \mathrm{~K}$ are found toward $\mathrm{MM}-1$, and along the ridge of $\mathrm{SiO}$ emission. In this high temperature region, there exists an area where the rotational temperatures cannot be derived. This is because the ratio of the $(1,1)$ and $(2,2)$ lines is sensitive to temperatures only up to $50 \mathrm{~K}$ (Ho \& Townes 1983). At temperatures over $50 \mathrm{~K}$, the ratio of the two lines approaches 1 for the optically thick case, and 1.3 in the optically thin case for a wide range of temperatures. A small error in the flux measurement will result in a large uncertainty 


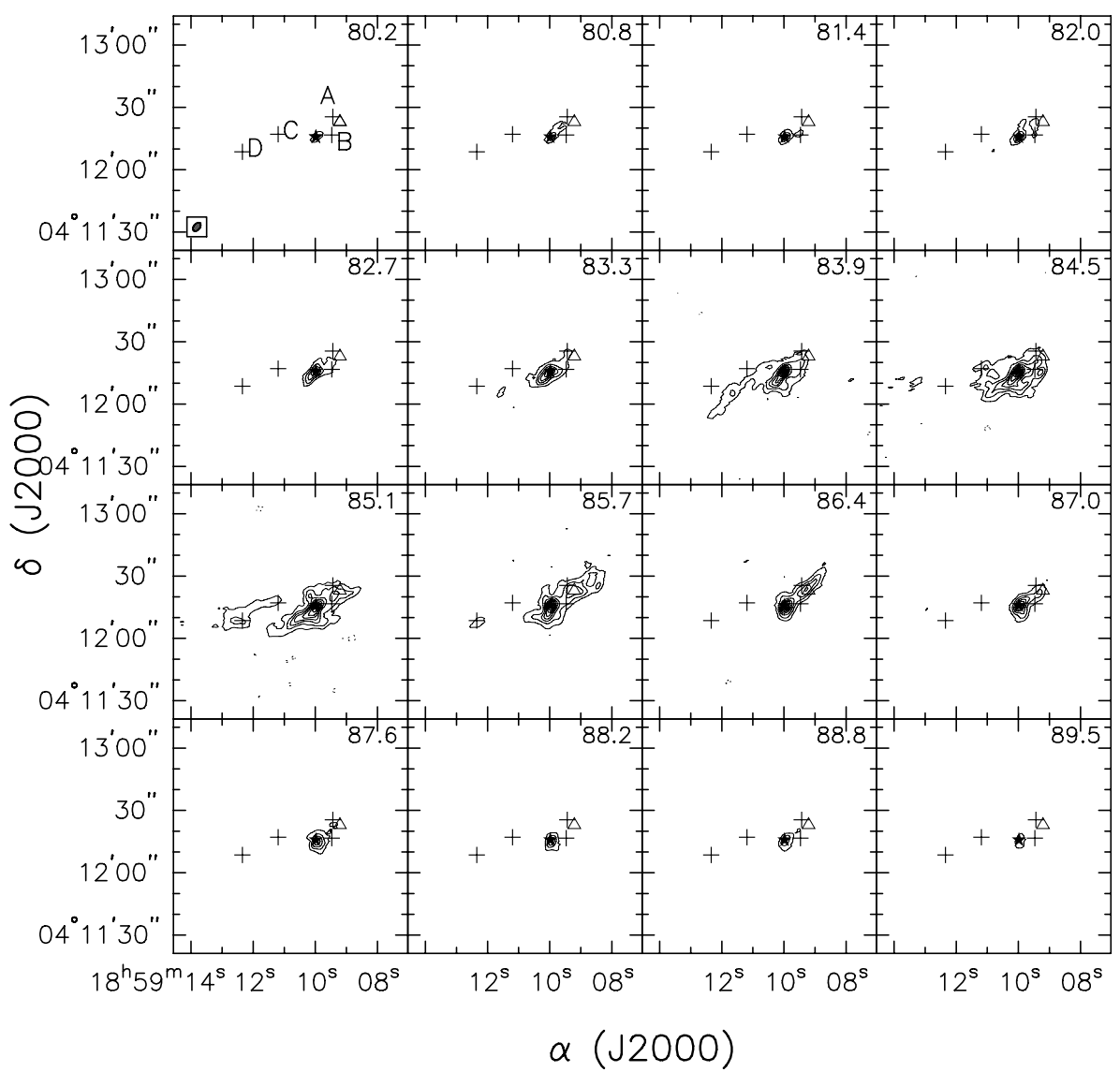

Fig. 4b. continued.

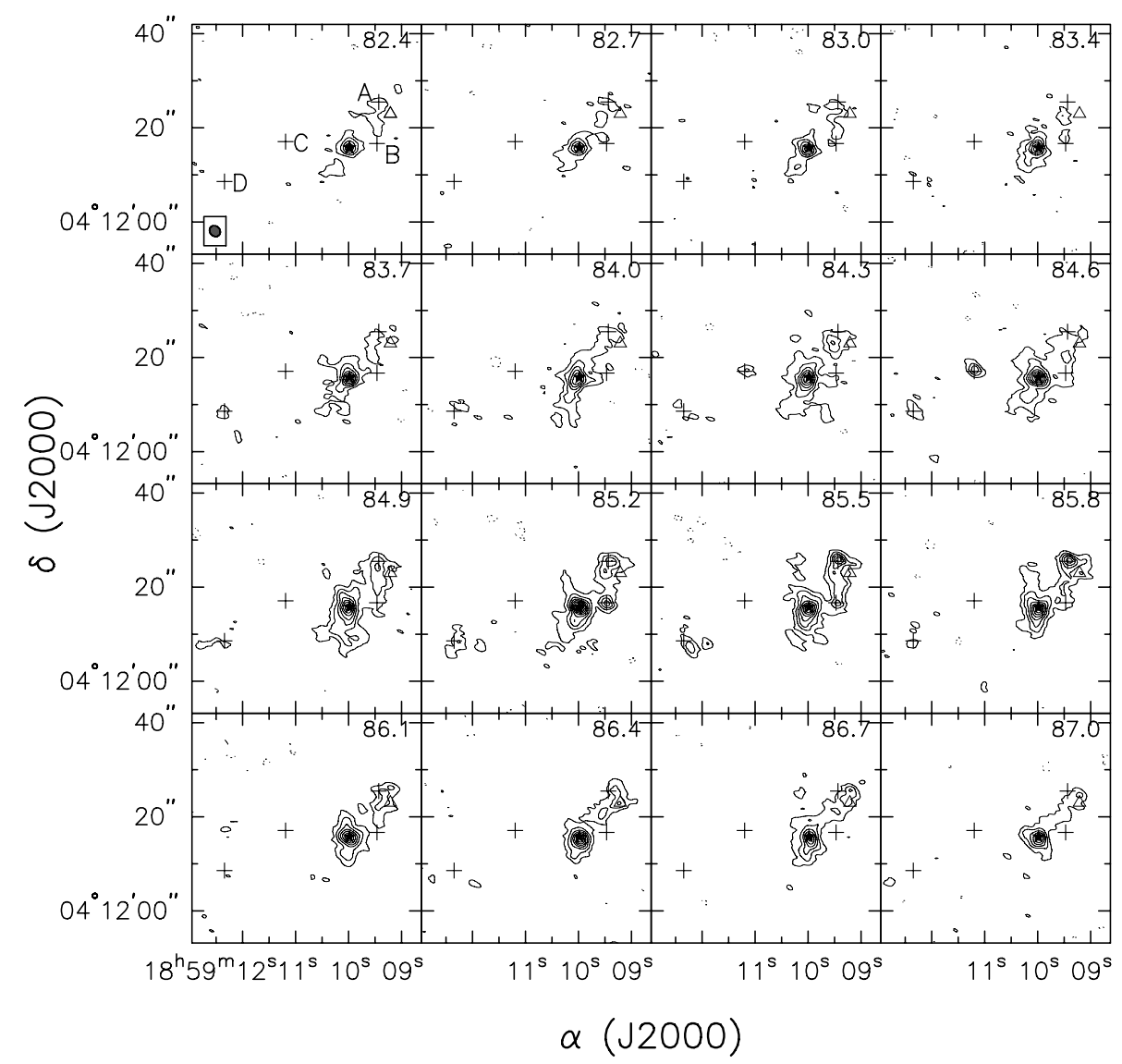

Fig. 4c. continued. 


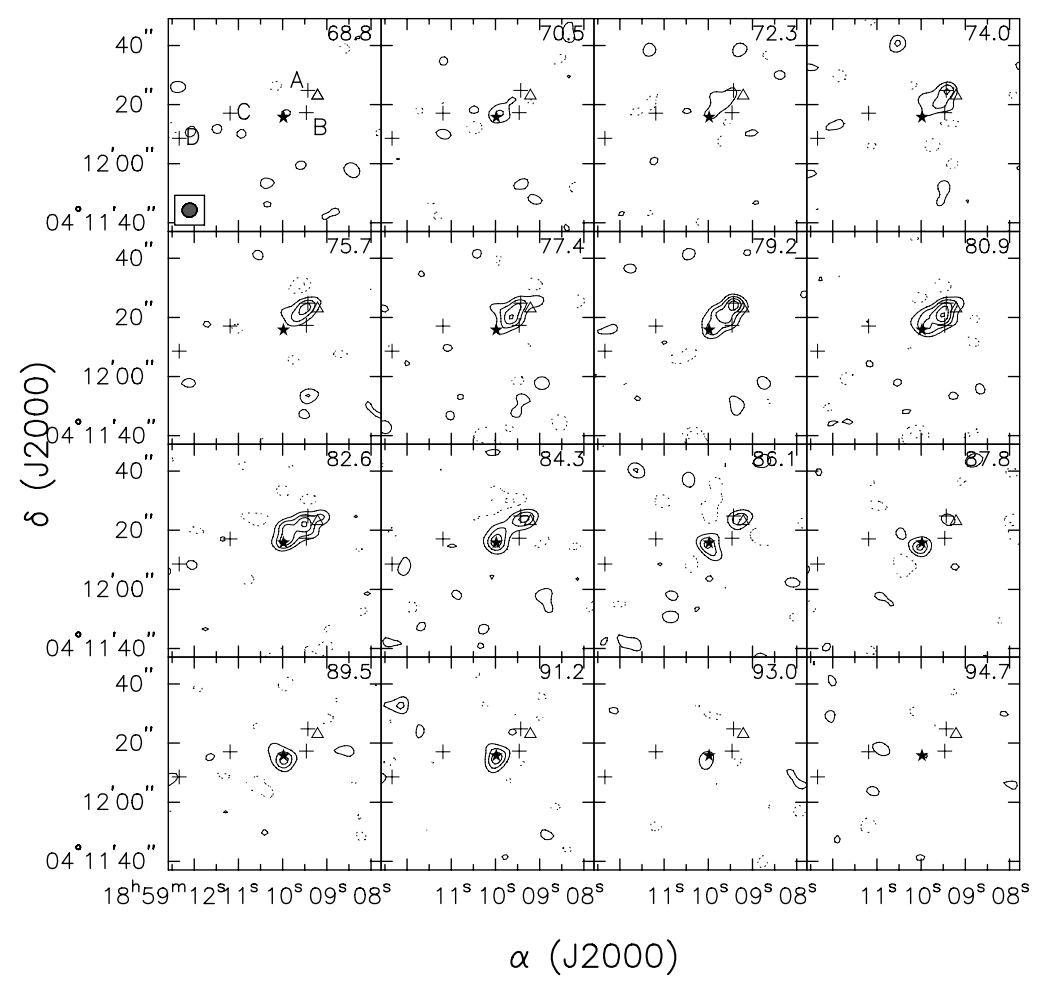

Fig. 5a. The channel maps of the $\mathrm{SiO} J=(2-1)$ (Fig. 5a) and $\mathrm{HCN} J=(1-0)$ (Fig. 5b) lines. The images were made using the OVRO E configuration data only. The contour levels are in steps of $0.05 \mathrm{Jy} / \mathrm{beam}$ for the SiO line, and $0.07 \mathrm{Jy} / \mathrm{beam}$ for the HCN line $(\sim 3 \sigma)$. The beam sizes are plotted at the lower-left of the first panel. The velocity of the channel is plotted at the upper-right of each panel. The "star" symbol and "triangle" mark the continuum peaks MM-1 and MM-2, respectively. The crosses mark the position of the hot $\mathrm{NH}_{3}(3,3)$ features.

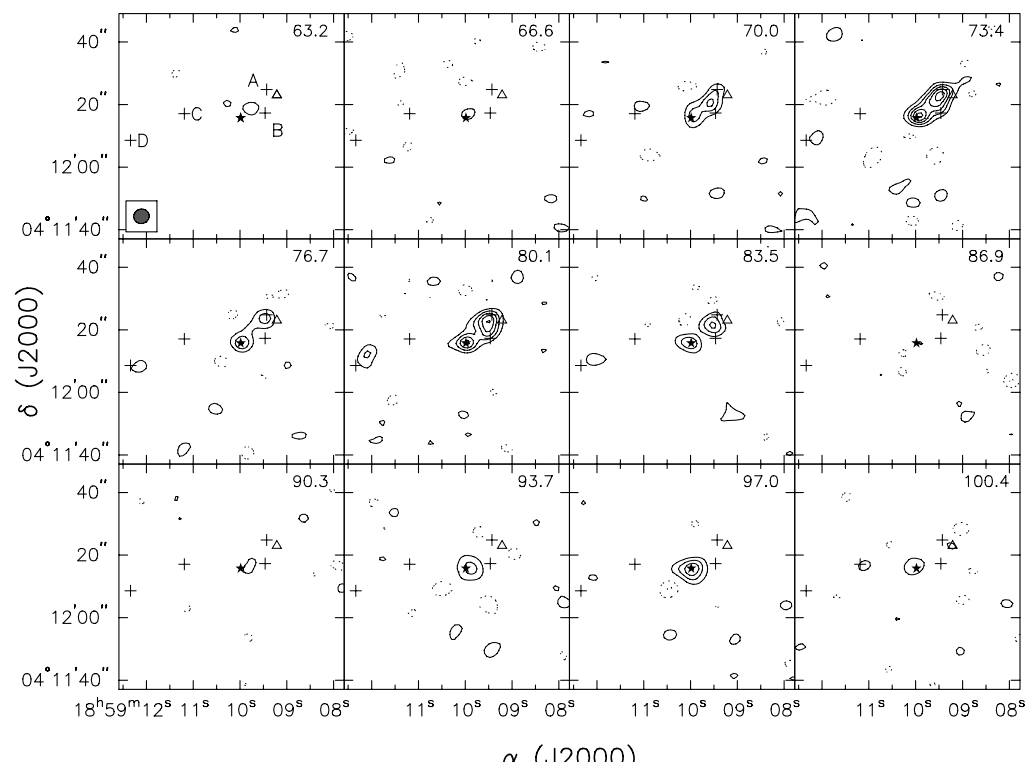

Fig. 5b. continued.

in rotational temperatures. Thus, the blanked area along the $\mathrm{SiO}$ outflow in Fig. 6 has even higher temperatures. Assuming the same abundance for the ortho and para $\mathrm{NH}_{3}$ species, we use the $(3,3)$ and the $(1,1)$ lines to obtain a temperature estimate of $70 \mathrm{~K}$ for blanked area in the outflow region.

\section{Discussions}

\subsection{Nature of the continuum peaks}

The $3 \mathrm{~mm}$ continuum emission reveals two peaks, MM-1 and MM-2, bridged by a faint extended filament. The dominant peak
MM- 1 coincides with the compact $7 \mathrm{~mm}$ continuum source, faint cm continuum emission (Araya et al. 2005; Carral et al. 1999), and the strong peaks in $\mathrm{NH}_{3}, \mathrm{SiO}$ and $\mathrm{HCN}$ emission. The rotational temperature estimated from $\mathrm{NH}_{3}$ is $45 \mathrm{~K}$, corresponding to a kinetic temperature of $80 \mathrm{~K}$ (Danby et al. 1988). The luminosity of the internal source required to produce the heating can be estimated by the following equation (Scoville \& Kwan 1976)

$$
T_{\mathrm{D}}=65\left(\frac{0.1 \mathrm{pc}}{r}\right)^{2 /(4+\beta)}\left(\frac{L_{\mathrm{star}}}{10^{5} L_{\odot}}\right)^{1 /(1+\beta)}\left(\frac{0.1}{f}\right)^{1 /(4+\beta)} \mathrm{K} .
$$




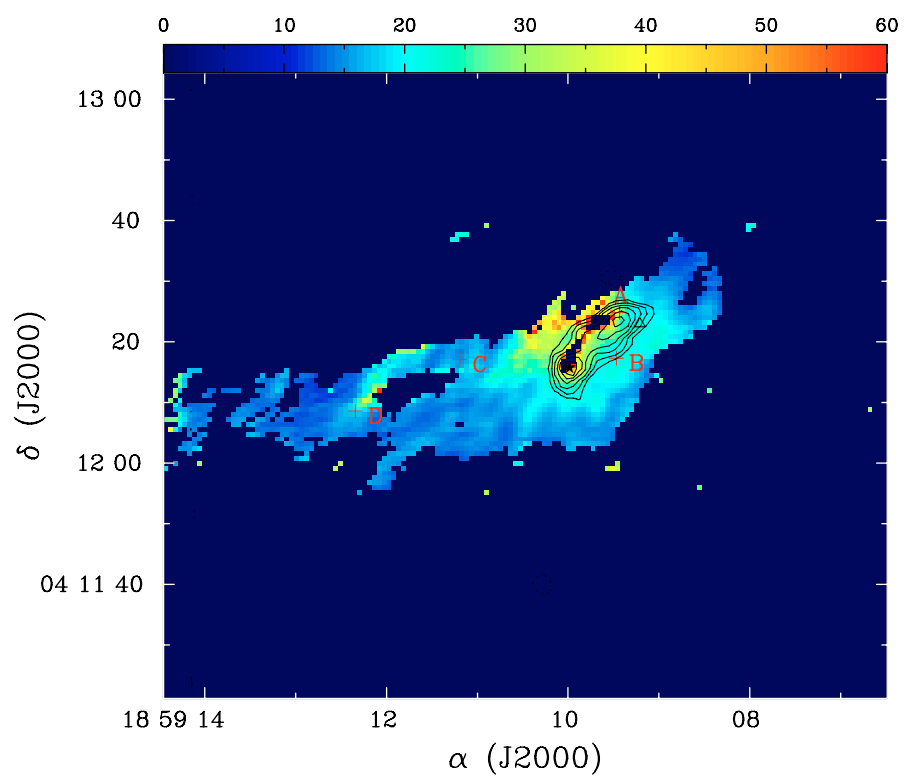

Fig. 6. The color scale shows the rotation temperatures derived from the $\mathrm{NH}_{3}(1,1)$ and $(2,2)$ lines. The contours represent the integrated emission of the SiO 2-1 line. The "star" symbol and "triangle" mark the continuum peaks MM-1 and MM-2, respectively. The crosses mark the position of the hot $\mathrm{NH}_{3}(3,3)$ features.

Here $\beta$ is the power law index of the dust emissivity at far infrared wavelengths, $f=0.08 \mathrm{~cm}^{2} \mathrm{~g}^{-1}$ is the value of the dust emissivity at $50 \mu \mathrm{m}$, and $r$ the core radius. If the dust and gas reach a thermal equilibrium in the high density environment, i.e., $T_{\mathrm{D}}=80 \mathrm{~K}$ at a radius $r=7000 \mathrm{AU}$, and $\beta=1$, we estimate the luminosity of the embedded source to be $6 \times 10^{4} L_{\odot}$. This value is in rough agreement with the far IR luminosity observed for the region. The high luminosity, strong dust and molecular line emission, and high temperature in the gas all indicate embedded massive protostar(s) toward MM-1.

The secondary mm peak MM-2 lies in a molecular ridge seen in $\mathrm{NH}_{3}$ and coincides with a local $\mathrm{NH}_{3}$ peak in the $(1,1)$ and $(2,2)$ transitions (see channels 85.7 and $86.4 \mathrm{~km} \mathrm{~s}^{-1}$ in Figs. $4 \mathrm{a}$ and $4 \mathrm{~b}$ ). This molecular ridge appears to correspond to the dust emission seen at $3 \mathrm{~mm}$ and $1.2 \mathrm{~mm} . \mathrm{NH}_{3}$ gas temperature toward MM-2 is about $30 \mathrm{~K}$. The large amount of dense molecular gas and a local peak in the gas and dust emission may indicate embedded protostar(s) in the core. If the heating of the gas and dust is due to an internal source, we find a luminosity of $8 \times 10^{3} L_{\odot}$ for the protostar, through a similar analysis as described above for MM-1. On the other hand, if the heating is partially due to the molecular outflow in the region (see discussions below), the total luminosity for MM-2 would be lower.

\subsection{Massive molecular outflow in $\mathrm{SiO}$ and $\mathrm{NH}_{3}$}

The SiO emission delineates a bipolar molecular outflow in the region. Figure 7 shows the position-velocity plots of the $\mathrm{SiO}$, $\mathrm{HCN}$, and $\mathrm{NH}_{3}(2,2)$ and $(3,3)$ emissions along the major axis of the $\mathrm{SiO}$ emission at a position angle of $135^{\circ}$. Toward the northwest of MM-1, the $\mathrm{SiO}$ emission is blue shifted with respect to the cloud systemic velocity of $85.2 \mathrm{~km} \mathrm{~s}^{-1}$. The terminal velocity of the blue-shifted $\mathrm{SiO}$ emission is about $15 \mathrm{~km} \mathrm{~s}^{-1}$ from the cloud velocity. Close to the peak MM-1, both the blue- and redshifted $\mathrm{SiO}$ emissions are detected up to $30 \mathrm{~km} \mathrm{~s}^{-1}$ ( $3 \sigma$ level) from the cloud velocity. The blue-shifted $\mathrm{SiO}$ emission extends $15^{\prime \prime}$ to the northwest. The red-shifted emission is far more compact spatially, with a peak detected only $2^{\prime \prime}$ southeast of MM-1.

As shown in Fig. 7, there exists cold and quiescent $\mathrm{NH}_{3}$ gas along the outflow direction. The $\mathrm{NH}_{3}$ emission peaks at $V_{\text {LSR }}$ of $85.2 \mathrm{~km} \mathrm{~s}^{-1}$, and has a narrow $F W H M$ of $<2 \mathrm{~km} \mathrm{~s}^{-1}$. In addition to the cold gas, there exists blue-shifted high velocity emission up to $V_{\mathrm{LSR}}$ of $70 \mathrm{~km} \mathrm{~s}^{-1}, 15 \mathrm{~km} \mathrm{~s}^{-1}$ from the cloud core velocity. This high velocity gas, offset to the northwest from the continuum peak, is part of the blue-shifted molecular outflow. The gas has an estimated temperature of $70 \mathrm{~K}$. Although $\mathrm{NH}_{3}$ is a reliable tracer of dense gas in molecular cloud cores, it can be affected by molecular outflows associated with both low and high mass stars (L1157: Tafalla \& Bachiller 1995; IRAS20126+4104: Zhang et al. 1999). The high velocity $\mathrm{NH}_{3}$ and $\mathrm{SiO}$ gas has been likely accelerated and heated by shock processes in the outflow.

Although the major axis of the $\mathrm{SiO}$ outflow agrees with that of the $\mathrm{CO} 2-1$ outflow obtained at $11^{\prime \prime}$ resolution (Beuther et al. 2002b), the CO outflow exhibits nearly symmetric bipolar morphology with the southeastern lobe much stronger than that in the $\mathrm{SiO}$. Furthermore, the polarity of the $\mathrm{SiO}$ outflow appears to be the opposite of that of the $\mathrm{CO}$ : the blue-shifted $\mathrm{SiO}$ emission lies to the northwest of the star, while the blue-shifted $\mathrm{CO}$ emission lies to the southeast of the star. This change of polarity between different tracers has been seen toward other objects (e.g. IRAS 20126+4104; Cesaroni et al. 1997; 1999). One plausible explanation is that the outflow axis lies almost in the plane of the sky and precesses. The low density CO gas traces the wide angle component in the outflow, while the high density $\mathrm{SiO}$ gas traces the well collimated jet component in the outflow. On the other hand, $\mathrm{CO}$ outflows toward massive star forming regions are often unresolved by single dish telescopes, and break into multiple bipolar outflows at high angular resolution (e.g. I05358, Beuther et al. 2002; AFGL 5142, Zhang et al. 2007). Furthermore, the $\mathrm{SiO}$ and $\mathrm{CO}$ may trace different outflows as shown in AFGL 5142 (Zhang et al. 2007; Hunter et al. 1999) and the Orion South region (Zapata et al. 2006). High resolution $\mathrm{CO}$ images of the outflow will help resolving the difference.

We compute the mass, momentum and energy in the SiO outflow. Using an $\mathrm{SiO}$ to $\mathrm{H}_{2}$ fractional abundance of $10^{-7}$ (Zhang et al. 1995), an excitation temperature of $70 \mathrm{~K}$ derived from $\mathrm{NH}_{3}$, and assuming optically thin $\mathrm{SiO}$ emission, we obtain outflow mass, momentum and energy of $18 M_{\odot}, 200 M_{\odot} \mathrm{km} \mathrm{s}^{-1}$, and $4.0 \times 10^{46} \mathrm{erg}$, respectively, in the blue-shifted lobe. Likewise, we obtain $9 M_{\odot}, 70 M_{\odot} \mathrm{km} \mathrm{s}^{-1}$, and $1.0 \times 10^{46} \mathrm{ergs}$ in the redshifted lobe. Despite the uncertainty in the $\mathrm{SiO}$ abundance, the total mass, momentum and energy of $27 M_{\odot}, 270 M_{\odot} \mathrm{km} \mathrm{s}^{-1}$, and $5.0 \times 10^{46} \mathrm{ergs}$ are in a rough agreement (within a factor of 2) with the estimates from the CO outflow (Beuther et al. 2002b).

The terminal velocity of $15 \mathrm{~km} \mathrm{~s}^{-1}$ and the length of the $\mathrm{SiO}$ outflow $\left(15^{\prime \prime}\right)$ yield a dynamical time scale $\left(T_{\text {dyn }}\right)$ of $1.7 \times$ $10^{4}$ years. This value is a few times smaller than that of the $\mathrm{CO}$ based on the lower angular resolution data (Beuther et al. 2002b). Assuming momentum conservation between the outflow and the underlying wind that powers the outflow: $m_{\mathrm{w}} V_{\mathrm{w}}=$ $P_{\text {outflow }}$, we can estimate the mass loss rate in the wind over the dynamical time scale of the outflow. The wind velocity $V_{\mathrm{w}}$ can vary from $100 \mathrm{~km} \mathrm{~s}^{-1}$ in low-mass stars to $500 \mathrm{~km} \mathrm{~s}^{-1}$ in highmass stars (Zhang et al. 2005). Since the effect of inclination angle of the outflow is not corrected, we use a lower value of $100 \mathrm{~km} \mathrm{~s}^{-1}$ for the wind velocity. This gives a mass loss rate $\left(m_{\mathrm{w}} / T_{\mathrm{dyn}}\right)$ of $1.5 \times 10^{-4} M_{\odot} \mathrm{yr}^{-1}$, and thus a lower limit to the mass accretion rate of $1.5 \times 10^{-4} M_{\odot} \mathrm{yr}^{-1}$, since some material presumably goes into the central protostar (Churchwell 2002). 

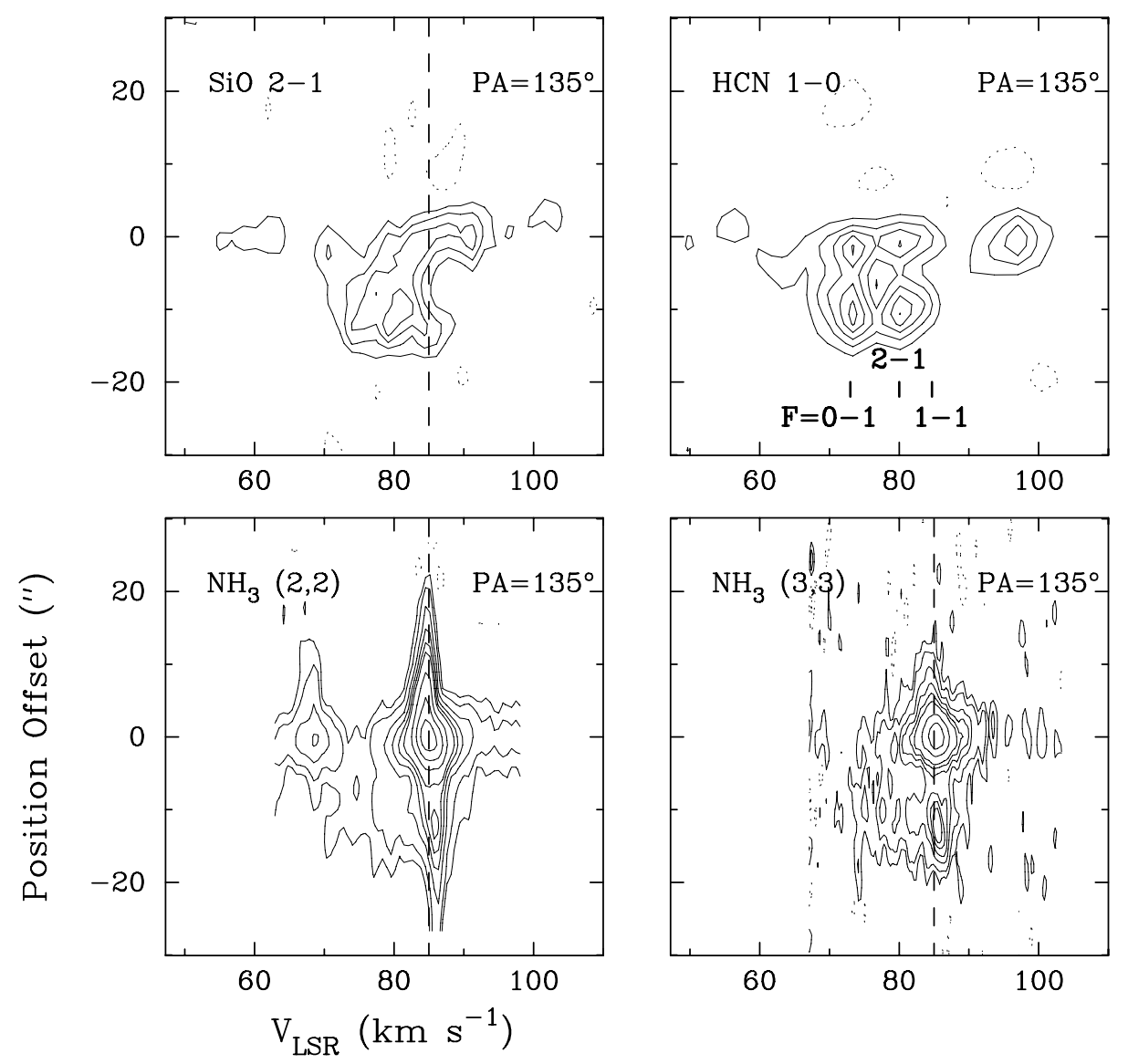

Fig. 7. The position-velocity plots of the $\mathrm{SiO} 2-1, \mathrm{HCN} 1-0, \mathrm{NH}_{3}(J, K)=(1,1),(2,2)$ and $(3,3)$ lines. The abcissa is offset from the position of the dust continuum with position angle (PA) of $135^{\circ}$. The positive offset represents the southeast of the dust continuum peak. The contours for the $\mathrm{SiO}$ and $\mathrm{HCN}$ are plotted in steps of $\pm 0.05 \mathrm{Jy} /$ beam for the $\mathrm{SiO}$ line, and $\pm 0.07 \mathrm{Jy} /$ beam for the $\mathrm{HCN}$ line $(\sim 3 \sigma)$, respectively. The $\mathrm{NH}_{3}$ data are contoured at $3 \times(1,2,4,6,8,10,15,20,25)$ mJy/beam. The three HCN hyperfine components are labeled assuming a cloud systemic velocity of $85.2 \mathrm{~km} \mathrm{~s}^{-1}$. The $\mathrm{NH}_{3}(2,2)$ emission around $72 \mathrm{~km} \mathrm{~s}^{-1}$ is the satellite hyperfine component.

\subsection{Heating and weak maser emission}

The outflow apparently causes significant heating in the molecular gas, especially toward the blue-shifted lobe. As shown in Fig. 6, the rotational temperature derived from the $\mathrm{NH}_{3}(1,1)$ and $(2,2)$ lines is $45 \mathrm{~K}$ toward the position of MM-1. However, the temperature in the outflowing gas toward the blue-shifted lobe is higher, with values of 50-70 K.

The effect of heating is further demonstrated in Fig. 8. We compute ratios of the $\mathrm{NH}_{3}(3,3)$ and $(1,1)$ emission. As the $(3,3)$ transition has an energy level of $124 \mathrm{~K}$, the higher ratios in general represent higher gas temperatures for thermal emission. As shown in Fig. 8, the $\mathrm{NH}_{3}$ gas in the outflow region exhibits consistently higher line ratios, as expected from high temperature regions. Toward the positions " $C$ " and " $D$ ", there appears to be heated $\mathrm{NH}_{3}$ gas in a bow shape. The $\mathrm{NH}_{3}$ spectrum toward "C" shows red-shifted line wings, while the $\mathrm{NH}_{3}$ spectra toward " $\mathrm{D}$ " show blue-shifted line wings. Furthermore, the tips of the bows point away from each other, as seen in the upper-right panel in Fig. 8. It is possible that the heating in $\mathrm{NH}_{3}$ traces another outflow, which is not seen in SiO. The heating likely arises from bow shocks as high velocity gas impinges on the cloud core. The potential driving source should lie in between " $C$ " and " $D$ ". However, no dust continuum emission is detected at a $3 \sigma$ limit of $4 M_{\odot}$.

Figure 9 presents $\mathrm{NH}_{3}$ spectra toward positions "A", "B", "C" and "D". All $\mathrm{NH}_{3}$ spectra display line wing emission
$15 \mathrm{~km} \mathrm{~s}^{-1}$ blue shifted from the cloud velocity. Toward "A", "B" and "C", the $(3,3)$ line has a $F W H M$ of $<1.5 \mathrm{~km} \mathrm{~s}^{-1}$, smaller than the $\sim 3 \mathrm{~km} \mathrm{~s}^{-1} F W H M$ in the $(1,1)$ and $(2,2)$ lines. The compact morphology, the narrow linewidth, and a lack of corresponding peaks in the $\mathrm{NH}_{3}(1,1)$ and $(2,2)$ emission indicate that the compact $(3,3)$ emission arises from population inversion, similar to maser emission. Maser inversion of the $\mathrm{NH}_{3}(3,3)$ has been detected toward a number of sources (e.g. W51, Zhang \& Ho 1995, NGC 6334, Kraemer \& Jackson 1995; Beuther et al. Beuther2007; DR 21(OH), Mangum \& Wootten 1994; Mauersberger et al. 1986; IRAS 20126+4104, Zhang et al. 2001). $\mathrm{NH}_{3}(3,3)$ inversion can form through collisional excitation of $\mathrm{NH}_{3}$ by $\mathrm{H}_{2}$ (Walmsley \& Ungerechts 1983). Through collisions with $\mathrm{H}_{2}$, the upper level of the $\mathrm{NH}_{3}(3,3)$ (denoted as $\left.\mathrm{NH}_{3}(3,3)^{+}\right)$exchanges with its $(0,0)$ state while the lower level of the $(3,3)$ exchanges with the $(1,0)$. Since the transition between the $(3,3)^{+}$and $(0,0)$ involves a change of parity and thus is more preferred, the $(3,3)^{+}$state can be overpopulated.

$\mathrm{NH}_{3}(3,3)$ masers are often observed in outflows. In the cases of IRAS 20126+4104 and NGC 6334 (Kraemer \& Jackson 1995; Zhang et al. 2001), (3,3) masers are detected in the vicinity of bow shocks where outflow wind interacts with the cloud gas. The high velocity $\mathrm{NH}_{3}$ gas detected toward "A", "B" and "C" (see Fig. 9) suggest a similar scenario. However, the spatially compact $\mathrm{NH}_{3}(3,3)$ emission appears to be resolved at an resolution of 1 ", suggesting that the emission is not strongly amplified. 

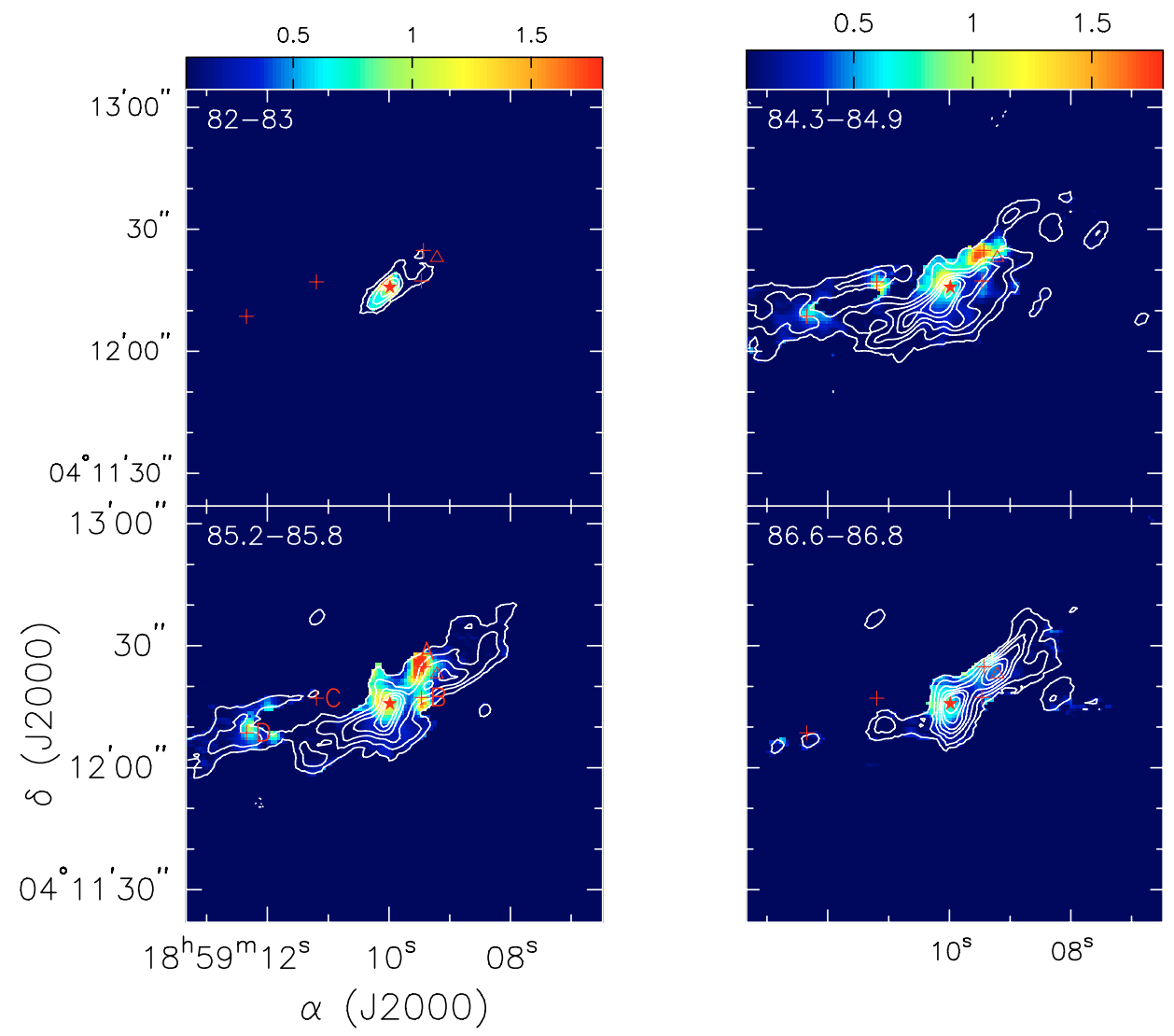

Fig. 8. The ratio of the integrated $\mathrm{NH}_{3}$ emission $(3,3) /(1,1)$ (color) overlaid on the integrated emission of the $\mathrm{NH}_{3}(J, K)=(1,1)$ line $($ contours). The velocity range of the integration in units of $\mathrm{km} \mathrm{s}^{-1}$ is given at the upper left corner of each panel. The "star" symbol and "triangle" mark the continuum peaks MM-1 and MM-2, respectively. The crosses marks the position of hot $\mathrm{NH}_{3}(3,3)$ features.

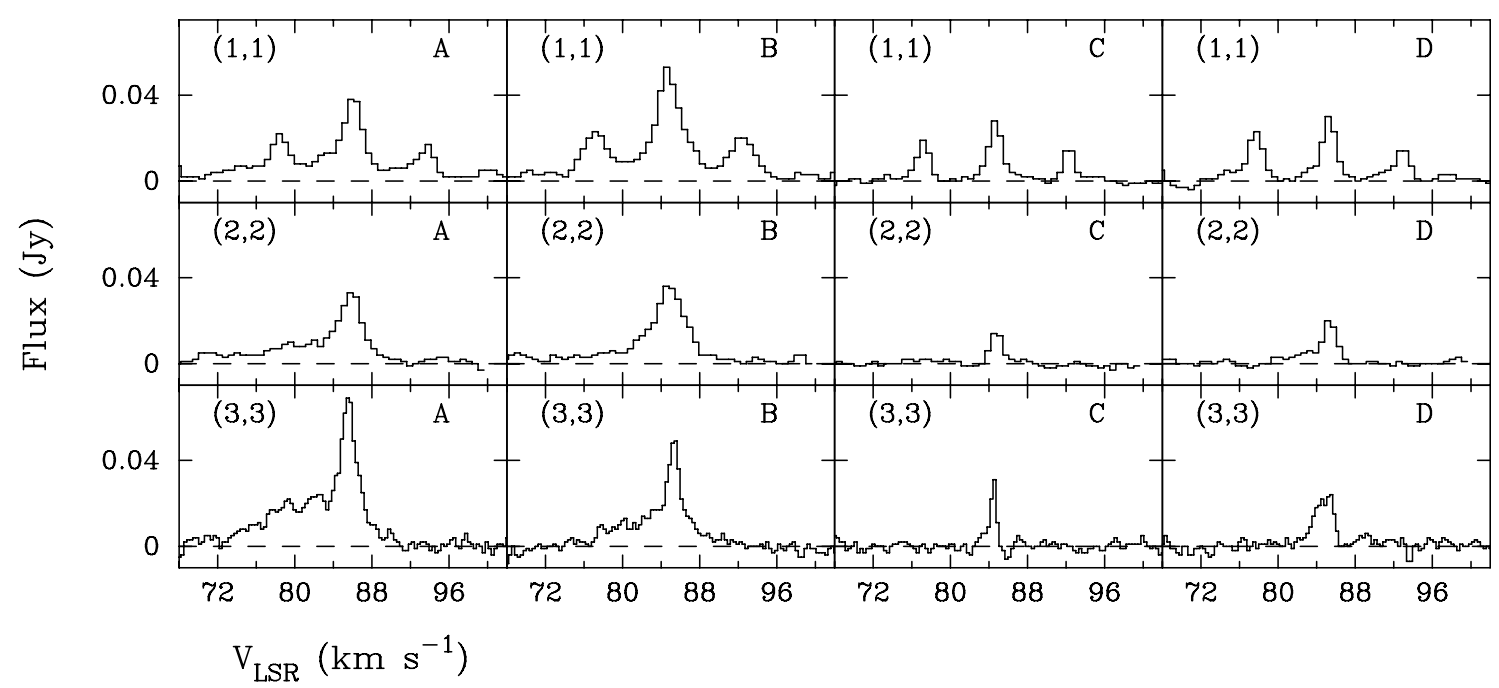

Fig. 9. Spectra of the $\mathrm{NH}_{3}(J, K)=(1,1),(2,2)$ and $(3,3)$ transitions from the compact components A, B, C, and D.

\subsection{Kinematics in the $\mathrm{NH}_{3}$ core}

In $\mathrm{MM}-1$, the compact $\mathrm{NH}_{3}$ emission with a Gaussian-like profile and large linewidth distinguishes itself from the relatively smooth $\mathrm{NH}_{3}$ emission in the cloud core. The $\mathrm{NH}_{3}$ emission toward this position has much broader linewidth: $5.5 \mathrm{~km} \mathrm{~s}^{-1}$ at a spatial resolution of $3^{\prime \prime}$. We image the visibility data from the VLA C array only and obtain an angular resolution of $1^{\prime \prime}$. From this image, we obtain a fitted $F W H M$ of $8.7 \mathrm{~km} \mathrm{~s}^{-1}$ in the $(3,3)$ line. This increase indicates additional broadening in the spectral lines towards the inner part of the $\mathrm{NH}_{3}$ core. The $\mathrm{NH}_{3}$ compact structure has a size of $1.2^{\prime \prime}$ or $8000 \mathrm{AU}$. We estimate the mass in this compact structure following Ho \& Townes (1983). With a rotational temperature of $45 \mathrm{~K}$, size of $1.2^{\prime \prime}$ and $\left[\frac{\mathrm{NH}_{3}}{\mathrm{H}_{2}}\right]=10^{-7}$ (Harju et al. 1993), and the assumption of LTE, we obtain a mass of $60 M_{\odot}$. This value is consistent with the mass estimated from the dust emission.

The broad $\mathrm{NH}_{3}$ linewidth toward MM-1 can arise from outflow, infall/rotation or relative motion of multiple objects 
unresolved within the synthesized beam. A collimated molecular outflow is present in the $\mathrm{SiO}$ emission with high velocity emission toward the northwest and the southest of MM-1. The effect of the outflow also appears in the $\mathrm{NH}_{3}$ emission, especially toward the northwest of MM-1 along the outflow lobe. Can the molecular outflow produce the line broadening seen in $\mathrm{NH}_{3}$ toward MM-1? The SiO emission is shifted from the cloud velocity. On the contrary, the $\mathrm{NH}_{3}$ emission peaks mostly at the cloud velocity and appears to be Gaussian in profile. Furthermore, the mass and momentum in the outflow within the 5 .' $^{\prime \prime} \times 4.4^{\prime \prime} 7$ area (the synthesized beam of the $\mathrm{SiO}$ data) of MM-1 are $1.5 M_{\odot}$ and $10 M_{\odot} \mathrm{km} \mathrm{s}^{-1}$, respectively. Similarly, we compute the same quantities from the $\mathrm{NH}_{3}$ gas over a scale of $1^{\prime \prime} .2$, and find the mass and momentum of $60 M_{\odot}$ and $250 M_{\odot} \mathrm{km} \mathrm{s}^{-1}$, respectively. The fractional abundances of $\mathrm{NH}_{3}$ and $\mathrm{SiO}$ may be uncertain and thus can affect the estimates provided above. Nevertheless, the comparison between the masses in $\mathrm{SiO}$ and $\mathrm{NH}_{3}$ shows that toward the most central region of the core only $<3 \%$ of the material traced by the $\mathrm{NH}_{3}$ emission is from the molecular outflow. Since the outflow mass is calculated over the area $\left(5^{\prime \prime} .0 \times 44^{\prime \prime} 7\right)$ 15 times larger than that of the $\mathrm{NH}_{3}$ emission, the actual contribution from the outflow can be even smaller. Thus, it is unlikely that the molecular outflow is the main contributor to the $\mathrm{NH}_{3}$ linewidth.

The remaining possibilities for the large $\mathrm{NH}_{3}$ linewidth are motions such as infall/rotation or relative motion of multiple cores within the synthesized beam. Higher angular resolution observations in dust continuum and spectral lines will be fruitful in distinguishing these possibilities. If the $\mathrm{NH}_{3}$ linewidths are due to rotation and infall, similar to the signature seen in $\mathrm{NH}_{3}$ toward IRAS 20126+4104 (Zhang et al. 1998), the dynamical mass, assuming gravitationally bound motion, derived using $M=\frac{V_{\mathrm{rot}}^{2} R}{2 G}$, is $35 M_{\odot}$, for $V_{\text {rot }}=3 \mathrm{~km} \mathrm{~s}^{-1}$ at $R=7000 \mathrm{AU}$. This is compatible with the mass estimate from dust emission and $\mathrm{NH}_{3}$ at a similar scale. The mass infall rate, estimated using $4 \pi R^{2} n_{\mathrm{H}_{2}} V_{\text {infall }}$, is $1.5 \times 10^{-3} M_{\odot} \mathrm{yr}^{-1}$ for $V_{\text {infall }}=3 \mathrm{~km} \mathrm{~s}^{-1}$ and $n_{\mathrm{H}_{2}}=10^{6} \mathrm{~cm}^{-3}$. Thus, the mass loss rate in the wind, $1.5 \times 10^{-4} M_{\odot} \mathrm{yr}^{-1}$ (Sect. 4.2), is $10 \%$ of the infall rate. Assuming that $10 \%$ to $30 \%$ of the infalling mass is ejected in the outflow, the accretion luminosity, estimated from $\frac{G M \dot{M}}{r}$, amounts to $3-4 \times 10^{4} L_{\odot}$, about half of the far-IR luminosity.

\section{Conclusion}

We conducted observations of the high-mass protostellar candidate IRAS $18566+0408$ with the VLA and OVRO interferometers.

(1) We resolve a collimated outflow in $\mathrm{SiO}$ (2-1) emission. The outflow is also detected in the line wings of $\mathrm{NH}_{3}$ inversion transitions and produces significant heating of the molecular gas (up to $70 \mathrm{~K}$ ). Compact features in the $\mathrm{NH}_{3}(3,3)$ line are detected along the outflow. The narrow linewidths of $<1.5 \mathrm{~km} \mathrm{~s}^{-1}$ suggest that they are weakly amplified maser emission.

(2) The $87 \mathrm{GHz}$ emission reveals two peaks MM-1 and MM-2. The internal heating in the MM- 1 core calls for embedded massive young star(s). The $\mathrm{NH}_{3}$ linewidth toward MM-1 is much broader than the typical linewidth of $<2 \mathrm{~km} \mathrm{~s}^{-1}$ in the extended core, and increases inward from $5.5 \mathrm{~km} \mathrm{~s}^{-1}$ at the $3^{\prime \prime}$ scale to $8.7 \mathrm{~km} \mathrm{~s}^{-1}$ at the $1^{\prime \prime}$ scale. The motion is consistent with rotation/infall, but can also arise from relative motions of unresolved protostellar cores.

Acknowledgements. We appreciate Editor M. Walmsley for his valuable comments. H. B. acknowledges financial support by the Emmy-Noether-Programm of the Deutsche Forschungsgemeinschaft (DFG, grant BE2578). Y. C. thanks the support by the NSFC Grant 110133020.

\section{References}

Araya, E., Hofner, P., Kurtz, S., et al. 2005, ApJ, 618, 339 Bachiller, R. 1996, ARA\&A, 34, 111

Bachiller, R., Martín-Pintado, J., \& Fuente, A. 1993, ApJ, 417, L45 Beltrán, M. T., Brand, J., Cesaroni, R., et al. 2006, A\&A, 447, 221 Beuther, H., Schilke, P., Menten, K. M., et al. 2002a, ApJ, 566, 945 Beuther, H., Schilke, P., Sridharan, T. K., et al. 2002b, A\&A, 383, 892 Beuther, H., Walsh, A., Schilke, P., et al. 2002c, A\&A, 390, 289 Beuther, H., Schilke, P., Gueth, F., et al. 2002d, A\&A, 387, 931 Beuther H., Hunter, T.R., Zhang, Q., et al. 2004, ApJ, 616, L23 Beuther, H., Zhang, Q., Sridharan, T. K., \& Chen Y. 2005, ApJ, 628, 800 Bronfman, L., Nyman, L.-A., \& May, J. 1996, ApJS, 71, 481 Burke, J. R., \& Hollenbach, D. J. 1983, ApJ, 265, 223 Carral, P., Kurtz, S., Rodríguez, L. F., et al. 1999, RMxAA, 35, 97 Cernicharo, J., Castets, A., Duvert, G., \& Guilloteau, S. 1984, A\&A, 139, L13 Cesaroni, R., Felli, M., Testi, L., Walmsley, C. M., \& Olmi, L. 1997, A\&A, 325, 725

Cesaroni, R., Felli, M., Jenness, T., et al. 1999, A\&A, 345, 949

Cesaroni, R., Neri, R., Olmi, L., et al. 2005, A\&A, 434, 1039

Churchwell, E. 2002, Hot Star Workshop III: The Earliest Phases of Massive Star Birth, 267, 3

Fontani, F., Beltrán, M. T., Brand, J., et al. 2005, A\&A, 432, 921

Gonzalez-Alfonso, E., \& Cernicharo, J. 1993, A\&A, 279, 506

Harju, J., Walmsley, C. M., \& Wouterloot, J. G. A. 1993, ApJS, 98, 51 Hildebrand, R. H. 1983, QJRAS, 24, 267

Ho, P. T. P., \& Townes, C. H. 1983, ARA\&A, 21, 239

Hunter, T. R., Testi, L., Zhang, Q., \& Sridharan, T. K. 1999, AJ, 118, 477

Kraemer, K. E., \& Jackson, J. M. 1995 ApJ, 439, L9

Mangum, J. G., \& Wootten, A. 1994, ApJ, 433, L134

Mauersberger, R., Wilson, T. L., \& Henkel, C. 1986, A\&A, 160, L13

McCutcheon, W. H., Dewdney, P. E., Purton, R., \& Sato, T. 1991, AJ, 101, 1435

Madden S. C., Irvine W. M., Matthews H. E., Brown R. D., \& Godfrey P. D. 1986, ApJ, 300, L79

Miralles, M. P., Rodríguez, L. F., \& Scalise, E. 1994, ApJS, 92, 173

Molinari, S., Brand, J., Cesaroni, R., \& Palla, F. 1996, A\&A, 308, 573

Osterloh, M., Henning, Th., \& Launhardt, R. 1997, ApJS, 110, 71

Pineau des Forêts, G., Flower, D. R., \& Chièze, J.-P. 1997, in Herbig-Haro Flows and the Birth of Low Mass Stars, ed. B. Reipurth, \& C. Dertout (Dordrecht: Kluwer), 199

Sako, S., Yamashita, T., Kataza, H., et al. 2005, Nature, 434, 995

Shepherd, D. S., \& Churchwell, E. 1996, ApJ, 472, 225

Shepherd, D.S., Claussen, M.J., \& Kurtz, S. 2001, Science, 292, 1513

Slysh, V. I., Val'tts, I. E., Kalenskii, S. V., et al. 1999, A\&AS, 134, 115

Scoville, N. Z., \& Kwan, J. 1976, ApJ, 206, 718

Sridharan, T. K., Beuther, H., Schilke, P., Menten, K. M., \& Wyrowski, F. 2002, ApJ, 566, 931

Walmsley, C. M., \& Ungerechts, H. 1983, A\&A, 122, 164

Walmsley, C. M., Churchwell, E., Nash, A., \& Fitzpatrick, E. 1982, ApJ, 258, L75

Williams, S. J., Fuller, G. A., \& Sridharan, T. K. 2004, A\&A, 417, 115

Wilking, B. A., Mundy, L. G., Blackwell, J. H., \& Howe, J. E. 1989, ApJ, 345, 257

Wu, J., \& Evans, N. J., II 2003, ApJ, 592, L79

Zapata, L. A., Rodríguez, L. F., Ho, P. T. P., et al. 2005, ApJ, 630, L85

Zhang, Q., \& Ho, P. T. P. 1995, ApJ, 450, L63

Zhang, Q., Hunter, T. R., \& Sridharan, T. K. 1998, ApJ, 505, L154

Zhang, Q., Hunter, T. R., Sridharan, T. K., \& Cesaroni, R. 1999, ApJ, 527, L117

Zhang, Q., Hunter, T. R., Brand, J., et al. 2001, ApJ, 552, L167

Zhang, Q., Hunter, T.R., Sridharan, T.K., \& Ho, P. T. P. 2002, ApJ, 566, 982

Zhang, Q., Hunter, T. R., Brand, J., et al. 2005, ApJ, 625, 864

Zhang, Q., Hunter, T. R., Beuther, H., et al. 2007, ApJ, 658, 1152

Ziurys, L. M., Friberg, P., \& Irvine, W. M. 1989, ApJ, 343, 201 